Revue d'histoire de l'Amérique française

REVUE D.HISTOIRE DE L'AMÉRIQUE FRANÇAISE

\title{
Documents uniques sur l'histoire du Québec
}

\section{Monique De la Roncière}

Volume 21, numéro 1, juin 1967

URI : https://id.erudit.org/iderudit/302649ar

DOI : https://doi.org/10.7202/302649ar

Aller au sommaire du numéro

Éditeur(s)

Institut d'histoire de l'Amérique française

ISSN

0035-2357 (imprimé)

1492-1383 (numérique)

Découvrir la revue

Citer ce document

De la Roncière, M. (1967). Documents uniques sur l'histoire du Québec. Revue d'histoire de l'Amérique française, 21(1), 118-124.

https://doi.org/10.7202/302649ar d'utilisation que vous pouvez consulter en ligne.

https://apropos.erudit.org/fr/usagers/politique-dutilisation/ 


\section{DOCUMENTS UNIQUES SUR L'HISTOIRE DU QUÉBEC}

$\mathrm{Au}$ sixième étage du Pavillon de la France, sous le signe de l'Amitié franco-canadienne, une exposition s'est donnée pour mission d'évoquer par des documents absolument authentiques, originaux - par conséquent presque toujours uniques - ce que pendant deux siècles et demi tant de Français, de Jacques Cartier à la fin du XVIIIe siècle, ont donné de leur force vive, de leur talent, de leur intelligence, de leur cœur, à ce pays immense alors mystérieux et si lointain qui les attirait pour de multiples raisons: fièvre de découvrir la route de l'ouest, rêve mystique, gloire maritime, soif d'aventure ou tout simplement la dure nécessité du pain quotidien. Aujourd'hui, plus de sept millions d'hommes descendent de ces pionniers. C'est à eux tout d'abord que nous présentons ce à quoi ils ont droit, et ce dont ils peuvent être fiers, ces souvenirs de leurs courageux ancêtres. Parmi la masse de documents existants, deux critères ont présidé à notre choix: n'apporter de France que des documents authentiques, et ne choisir que les plus beaux. Cette exposition n'a donc pas la prétention de faire œuvre historique exhaustive, mais de diriger sur certains personnages, certains événements, la lueur des projecteurs. Parmi les cent soixante-cinq documents ou objets exposés nous ne pouvons malheureusement ici n'en citer que quelquesuns: un magnifique globe de la Renaissance (globe dit de Rouen), supporté par quatre tritons de bronze dont la gravure sur cuivre indique la connaissance que l'on avait des côtes américaines entre Verrazzano et Cartier; la relation manuscrite du deuxième voyage de J. Cartier sous une reliure aux armes du roi Charles IX; le somptueux recueil de cartes enluminées du normand Guillaume Le Testu dédié en 1556 à l'amiral de Coligny, et dont la consultation attentive a permis dernièrement la reconstitution de la "Grande Hermine"; la première lettre datée du Canada (1542) et signée Roberval; une peinture bien émouvante et belle de saint Isaac Jogues et représentant son martyre; les cartes de l'ingénieur hydrographe J.-B.-L. Franquelin qui fit œuvre exquise de paysagiste; le premier plan de Montréal avec la liste en 1702 de plus de quatre cents familles; le plan cadastral de 
plus de huit mètres de long levé par Decoüagne et Catalogne indiquant toutes les propriétés riveraines du Saint-Laurent; des aquarelles de l'Ile-du-Cap-Breton, y compris Louisbourg; les beaux lavis de la Mission de Meules en Acadie et Gaspésie dont les teintes très vives semblent déjà annoncer nos impressionnistes français. Nous souhaitons ardemment, par cette énumération bien incomplète, avoir éveillé chez beaucoup le désir de venir se plonger dans le passé de leur pays.

MONIQUE DE LA RONCIÈRE conservateur à la Bibliothèque nationale de Paris, France

\section{LISTE DES DOCUMENTS}

\section{I \\ DÉCOUVREURS ET PREMIERS COLONS}

1. - Globe terrestre $\mathrm{XVI}^{\circ}$ s., dit Globe de Rouen.

2. - Manoir d'Ango. XVI ${ }^{\circ}$ s. Photographie.

3. - La Nouvelle France de Gastaldi. 1556.

4. - Portrait de Jacques Cartier. Burin de P. Gandon, 1934.

5. - Journal manuscrit du $2^{\circ}$ voyage de J. Cartier. Reliure aux armes de Charles IX.

6. - Vue d'Hochelaga in Ramusio. $\mathrm{XVI}^{\circ} \mathrm{s}$.

7. - Reliure $\mathrm{XVI}^{\circ}$ s. à décor de Peaux-Rouges.

8. - Limoëlou, manoir de J. Cartier. Photographie.

9. - Première lettre écrite du Canada. 9 sept. 1542 et signée Roberval.

10. - Portrait de Roberval, dessin de Clouet. Photographie.

11. - La Cosmographie de Jean Alfonce (c. 1545). 1 recueil manuscrit avec tracés de côtes.

12. - Cosmographie universelle de G. Le Testu. 1556. 1 album d'enluminures.

13. - Les Singularitez de la France Antarctique, par A. Thevet. 1558.

14. - Planisphère de Desliens. 1566. Peinture sur vélin.

15. - Sceau de P. Desceliers. XVI ${ }^{\circ}$ s.

16. - Les Grandes œuvres de J. de Vaulx. 1584.

17. - Brevis Narratio rerum quæ in Florida... Gallis acciderunt. Francofurti, Th. de Bry, 1591. Neuf facsimilés tirés de la "Floride Française" de Ch. de La Roncière (1928), accompagnent l'original.

18. - Vitrail normand de la fin du $\mathrm{XVI}^{\circ}$ s. Copie.

19. - La Sainte Galiote, gravure de G. David. Fin XVI ${ }^{\circ} \mathrm{s}$.

20. - Carte de l'Atlantique, par Pierre Devaulx. 1613, portulan enluminé.

21. - Boussole-cadran, de Ch. Bloud. Début XVII ${ }^{\circ} \mathrm{s}$.

22. - Histoire de la Nouvelle France par M. Lescarbot, 1609. Reliure aux armes de Louis XIII dauphin.

23. - Honfleur, lavis du XVII ${ }^{\circ} \mathrm{s}$.

24. - Québec in Voyages du Sieur Champlain, 1613. 
25. - Voyages de Champlain. - Paris, Cl. Collet, 1632.

26. - Brouage, grav. de $\mathrm{Cl}$. Chastillon. XVII ${ }^{\circ} \mathrm{s}$.

27. - Carte Universelle... faitte par Jean Guérard. Peinture sur vélin. 1634.

28. - Vue de Mortagne, grav. Milieu XVII ${ }^{\circ} \mathrm{s}$.

29. - Histoire véritable... des mœurs de la Nouvelle France, par P. Boucher. 1664.

30. - Carte manuscrite de Jehan Bourdon. 1641. Dessin à la plume.

31. - Vie de M. Marie de l'Incarnation. 1667, par Dom G. Martin.

32. - Portrait de M. Marie de l'Incarnation, gravure de Poilly.

33. - Vue de Tours, gravure de J. Boisseau. 1643.

34. - Vue à vol d'oiseau de l'île d'Orléans, lavis (c. 1670).

35. - Deux lettres des religieuses de l'Hôtel-Dieu de Québec. 1734-1738.

36. - Deux Pots de pharmacie en vieux-Rouen, XVIII $\mathrm{s}$.

37. - La Nouvelle France, vers 1660 . Vélin colorié au lavis.

38. - Le Renard, dessin colorié. XVII ${ }^{\circ}$ s.

39. - L'Hermine, dessin colorié. XVII ${ }^{\circ}$ s.

\section{II}

\section{MARTYRS JÉSUITES}

40. - Relation manuscrite du P. Paul Le Jeune. 1634.

41. - Relation de ce qui s'est passé en la Nouvelle France en l'année 1639, du P. Paul Le Jeune. Paris, S. Cramoisy, 1640. Reliure aux armes du Grand Condé.

42. - Lettre autographe de St Jean de Brébeuf. 1634, écrite sur le chemin de "Trois Rivières".

43. - Novæ Franciæ... Delineatio. 1657. Avec cartouche représentant martyre des P. Brébeuf et G. Lalemant.

44. - De l'heureuse mort du P. Jean de Brébeuf et du P. Gabriel Lallement, relation manuscrite du $P$. Ragueneau.

45. - Portrait de St Isaac Jogues. Peinture française du XVII ${ }^{\circ} \mathrm{s}$.

46. - Tableau du martyre de St Isaac Jogues. Peinture française du $\mathrm{XVII}{ }^{\circ} \mathrm{s}$.

47. - Lac Supérieur et autres lieux où sont les missions des Pères de la Cie de Jésus. 1672. Carte gravée.

48. - Pirogue indienne montée par cinq personnages. Ex-voto fin $\mathrm{XVII}^{\circ} \mathrm{s}$. provenant de la cathédrale de Chartres.

\section{III \\ MONTREAL}

Fondation - Paix iroquoise - Implantation humaine

49. - Minute autographe du Contrat des Associés de Montréal. 1641.

50. - J. J. Olier. Miniature sur vélin de $\mathrm{H}$. Strésor. XVII ${ }^{\circ} \mathrm{s}$.

51. - La Dauversière. Portrait à l'huile. XVII ${ }^{\circ}$ s.

52. - Relation de ce qui s'est passé en la Nouvelle France en l'année 1642 ... Par le R. P. Vimont. 
53. - Lettre autographe de Marguerite Bourgeois, de Ville Marie. 1695.

54. - Plan manuscrit du Quartier de St Jean à Troyes. XVII ${ }^{\circ}$ s.

55. - Le Colombier de Chomedey de Maisonneuve. Photographie.

56. - Relation manuscrite des faits héroïques de M.M. de Verchères. 22 oct. 1696.

57. - Paroles des Iroquois et réponses à M. de Callières. Signatures idéogrammatiques. Manuscrit.

58. - Catéchisme du Diocèse de Québec, par Mgr. de Saint Valier. Paris, 1702.

59. - Description généralle de l'Isle de Montréal... en 1702 avec le nom de chaque habitant et la quantité de terre qu'ils possèdent. Manuscrit.

60. - Plan de Montréal par Decouagne. 1749. Manuscrit rehaussé de couleurs.

61. - Relation manuscrite d'un voyage de Paris à Montréal en 1737, par M. J. Dargent.

\section{IV}

\section{COLBERT, LE MINISTRE DE LA MARINE}

et

\section{J.-B.-L. FRANQUELIN, L'INGÉNIEUR HYDROGRAPHE}

62. - Portrait de Colbert, aquarelle sur vélin. XVII ${ }^{\circ} \mathrm{s}$.

63. - Desseins des différentes manières de vaisseaux... Album manuscrit relié de maroquin rouge aux armes de Colbert.

64. - Carte du Saint Laurent de J. B. L. Franquelin. 1678. Dessin à la plume.

65. - Carte de la mine d'argent. Dessin à la plume.

66. - Le Fort St Louis de Québec, par J. B. L. Franquelin. 1683. Dessin à la plume.

67. - Carte de l'Amérique septentrionale de J. B. L. Franquelin, ornée d'une vue de Québec. 1688. Dessin rehaussé de lavis.

68. - Carte de la Nouvelle France. 1702. Manuscrit sur vélin rehaussé de lavis.

69. - Rivière de Saguenay avec deux vues de Chekoutimi vers 1748. Dessin colorié.

\section{V}

\section{L'ACADIE}

\section{D'ISAAC DE RAZILLY Ã L'INTENDANT DE MEULLES}

70. - Autographe du Cardinal de Richelieu, au bas d'instructions données à Razilly. 1629.

71. - Lettres patentes de Louis XIII. 20 avril 1632, nommant Razilly gouverneur de la Nouvelle France.

72. - Psautier de 1591 ayant appartenu à Isaac de Razilly.

73. - Partie de la Coste d'Acadie. Carte manuscrite sur vélin ayant appartenu à Claude de Razilly, Sieur de Launay. 1632.

74. - Atlas maritime de Jacob Aertsz Colom, 1642, portant la signature de Claude de Razilly, Sieur de Launay. 
75. - Havre de la Haive. Dessin au lavis levé au cours de la mission de Meules. 1685.

76. - Plan de Port Royal. Dessin au lavis, levé au cours de la mission de Meules. 1685.

77. - Rade de l'Isle Percée. Dessin au lavis levé au cours de la mission de Meules. 1685.

78. - Boussole, ou Compas de mer, fait par Ch. Picard à St Malo vers 1650 .

\section{VI}

\section{LA ROUTE DE L'OUEST AUX XVIIe ET XVIIIe S.}

79. - Carte manuscrite coloriée dite du Père Marquette. 1673.

80. - Relation manuscrite du voyage du P. Marquette.

81. - Carte manuscrite de la découverte du Sr. Jolliet, dédiée à Frontenac. 1673.

82. - Voyage de M. de Salle, relation manuscrite signée Minet.

83. - Lettre signée d'Iberville. 1699.

84. - Journal du voyage fait par d'Iberville à la coste méridionale de Floride en 1699. Manuscrit.

85. - Plan manuscrit du Fort Condé de la Mobille. 1725, par De Pauges.

86. - Plan manuscrit colorié de la Nouvelle Orléans. (c. 1730).

87. - Le Fort Rosalie des Natchez ... manuscrit 1729.

88. - Le Fort Chambly, plan manuscrit de Beaucours. 1710.

89. - Plan du Fort du Détroit, dessiné par Chaussegros de Léry. 1749.

90. - Carte de La Vérendry, des rivières et fleuves courant à l'Ouest. Manuscrit.

91. - Aventure du Sr. Le Beau. 2 vol. 1738.

92. - M. de La Galissonnière, grav. par Hubert.

93. - Mœurs des sauvages amériquains... Par le Père Lafitau. 2 vol. $4^{\circ}$, reliure aux armes de la Comtesse de Provence.

94. - La Tourterelle du Canada in "Histoire naturelle des Oiseaux" de Buffon. 1770, 1 pl. col.

95. - Le Lynx du Canada in "Animaux quadrupèdes" de Buffon, pl. coloriée.

96. - Le Castor in "Animaux quadrupèdes" de Buffon, pl. coloriée.

97. - Le Canard à Collier de Terre Neuve in L" "Histoire naturelle des Oiseaux" de Buffon, pl. coloriée.

98. - Danse d'Indiens, dessin. XVIII ${ }^{\circ}$ s., attribué à G. de Saint-Aubin.

99. - Vue du Sault du Niagara. Lavis de la fin du XVIII ${ }^{\circ} \mathrm{s}$.

100. - Description des chûtes de Niagara, in Mémoires d'Outre-tombe de Chateaubriand.

101. - Baïonnette de chasse. XVII ${ }^{\circ} \mathrm{s}$.

102. - Portefeuille en maroquin rouge. $\mathrm{XVIII}^{\circ} \mathrm{s}$.

\section{VII}

\section{LOUISBOURG}

103. - Vue du Port Dauphin. Aquarelle. 1717.

104. - Vue du Port Toulouse. Aquarelle. 1717. 
105. - Vue du Port Louisbourg. Aquarelle. 1717.

106. - Vue de la ville de Louisbourg, par Verrier. 1731. Dessin au lavis.

107. - Plan de Louisbourg, par Verrier. 1745. Dessin au lavis.

108. - Frégate XVIII ${ }^{\circ}$ s. Maquette.

109. - Les Aventures de M. Robert Chevalier dit de Beauchêne, par Le Sage, 2 vol. in $-8^{\circ}$.

VIII

\section{TERRE-NEUVE}

110. - Carte basque de l'Atlantique faicte à St Jean de Luz, par Denis de Rotis 1674. Portulan peint.

111. - Voyage aventureux du Capitaine Martin de Hoyarsabal, routier basque, éd. de 1633.

112. - Plan géométral et Fort de Plaisance, par Thomas Bonaventure Godalles. 1712. 1 flle manuscrite coloriée.

113. - La Louise de Canada. Peinture à l'huile (c. 1730) Ex-voto provenant de la cathédrale de La Rochelle.

114. - Vue du Chapeau Rouge. Dessin ms. au lavis. XVIII ${ }^{\circ} \mathrm{s}$.

115. - Plan et élévation du Fort Pontchartrain à la coste de Labrador. Dessin à la plume par Beaucours. 1708.

116. - Plan de l'habitation de Charles Mahier à Plaisance en 1714.

117. - Quatre gravures tirées du "Traité général des pêches" par M. Duhamel du Monceau. Photographies.

118. - Kayac esquimau en peau de phoque. XVIII ${ }^{\circ}$ s. provenant du musée ethnographique du Roi. Versailles.

119. - Voyage fait par ordre du Roi en 1750 et 1751 dans l'Amérique Septentrionale... par Chabert. 1753. Reliure aux armes du Dauphin.

120. - Arbalestrille du XVIII ${ }^{\circ}$ s.

121. - Pochette de mathématiques. XVIII ${ }^{\circ} \mathrm{s}$.

122. - Echelle de réduction en cristal, par R. Fuess.

123. - Compas à pointe sèche du milieu XVIII ${ }^{\circ} \mathrm{s}$.

124. - Compas de Galilée, par Canivet. XVIII ${ }^{\circ} \mathrm{s}$.

125. - Règlette graduée en ivoire, divisée en pouces. XVIII ${ }^{\circ} \mathrm{s}$.

IX

\section{MONTCALM - BOUGAINVILLE - LA PEROUSE}

126. - Portrait au crayon du Général de Montcalm. XVIII ${ }^{\circ} \mathrm{s}$.

127. - Miniature du XVIII ${ }^{\circ}$ s. représentant le Général de Montcalm.

128. - Morceau de rocher sur lequel fut blessé Montcalm, surmonté d'un camée.

129. - Mercure de France. 1760. Reliure maroq. rouge aux armes royales.

130. - Bonbonnière en écaille de la fin du XVIII ${ }^{\circ} \mathrm{s}$. avec miniature représentant Montcalm mortellement blessé.

131. - Jolie miniature de la fin du XVIII ${ }^{\circ}$ s. représentant Jeanne-Marie de Lévis.

132. - Soldats de plomb. XVIII ${ }^{\circ}$ s.

133. - Portrait de Louis Jean Pierre Marie Gilbert de Montcalm. Peinture à l'huile XVIII ${ }^{\circ}$ s. Fils du Général de Montcalm. 
134. - Portrait de François Déodat Gilbert de Montcalm. Peinture à l'huile. $\mathrm{XVIII}^{\circ}$ s. Fils cadet du général de Montcalm.

135. - Miniature de Louis-Antoine de Bougainville attribuée à Isabey.

136. - La Pérousse, grav. de la seconde moitié du XVIII ${ }^{\circ} \mathrm{s}$.

137. - Vue du Fort du Prince Walles, par Lefebvre. Dessin rehaussé de lavis, fin XVIII ${ }^{\circ} \mathrm{s}$.

138. - Vue du Fort d'York par Lefebvre. Dessin rehaussé de lavis.

139. - Boussole XVIII ${ }^{\circ}$ s. boîtier ivoire, objet personnel de La Pérouse.

140. - Tabatière XVIII ${ }^{\circ}$ s., objet personnel de La Pérouse.

\section{$\mathbf{X}$}

\section{LE GRAND INVENTAIRE DES PROPRIÉTÉS DU QUEBEC}

141. - Cinq cartes du gouvernement de Québec et de Trois Rivières assemblées en 1 flle de $8 \mathrm{~m} .350$ (long) $\mathrm{x} 1 \mathrm{~m} .200$ (haut).

\section{$\mathrm{XI}$}

\section{DES PORTS DE FRANCE À QUÉBEC}

142. - Carte des côtes de France, par Jean Guerard. 1627. Portulan enluminé aux armes de Jean de Lauson.

143. - Le Pourtraict de la ville de Dieppe. 1575. Grav. sur bois.

144. - Rouen, vue grav. par Jollain, $\mathrm{XVII}^{\circ} \mathrm{s}$.

145. - Le Port de Fécamps, dessin d'Ozanne. 1776.

146. - Saint Malo, dessin au lavis. $\mathrm{XVII}^{\circ} \mathrm{s}$.

147. - Vue d'optique. XVIII ${ }^{\circ}$ s. St Malo.

148. - Vue d'optique. XVIII ${ }^{\circ}$ s. Rouen.

149. - Vue d'optique d'un embarquement à Brest. XVIII ${ }^{\circ} \mathrm{s}$.

150. - Nantes, grav. du milieu du XVII ${ }^{\circ} \mathrm{s}$.

151. - La Rochelle, très beau dessin au lavis de la fin du XVII ${ }^{\circ} \mathrm{s}$.

152. - Bordeaux. XVII ${ }^{\circ}$ s.

153. - Vue d'optique. Bordeaux. XVIII ${ }^{\circ}$ s.

154. - Saint Jean de Luz, plan manuscrit en couleurs sur vélin. XVII ${ }^{\circ} \mathrm{s}$.

155. - Vue d'optique. Bayonne, XVIII ${ }^{\circ}$ s.

156. - Dessin de Mahier 1729. Très jolie vue de Québec.

157. - Les Forges du Roy à Québec, dessin colorié par Chaussegros de Léry. 1749.

158. - Tours bastionnées de Québec. 1710. Dessin à la plume.

159. - La redoute du moulin à Québec, par Beaucours. Dessin à la plume.

160. - Elévation du perron ou terrasse du nouveau palais de l'Intendant. Dessin à la plume. XVIII ${ }^{\circ} \mathrm{s}$.

161. - Vue au lavis de la ville de Trois Rivières. XVIII ${ }^{\circ} \mathrm{s}$.

162. - Vue d'optique. XVIII ${ }^{\circ}$ s. Haute ville de Québec.

163. - Vue d'optique. XVIII ${ }^{\circ}$ s. Basse ville de Québec.

164. - Vue d'optique. XVIII ${ }^{\circ}$ s. Rue des Récollets, Québec. 